\title{
REPENSANDO LOS DERECHOS CONSTITUCIONALES DE LA PERSONA JURÍDICA A LA LUZ DEL OBAMACARE
}

\author{
Re-thinking the corporation's constitutional rights \\ in the light of the Obamacare
}

\author{
ANA MARÍA RUIZ GONZÁLEZ \\ Universidad de Navarra \\ aruiz.46@alumni.unav.es \\ Cómo citar/Citation \\ Ruiz González, A. M. (2018). \\ Repensando los derechos constitucionales \\ de la persona jurídica a la luz del Obamacare. \\ Revista de Estudios Políticos, 181, 187-216. \\ doi: https://doi.org/10.18042/cepc/rep.181.07
}

\section{Resumen}

La titularidad de la libertad religiosa para las corporaciones con ánimo de lucro fue el tema central discutido en el emblemático caso de la Corte Suprema de Estados Unidos Burwell v. Hobby Lobby. En un ajustado fallo de cinco votos contra cuatro, la opinión mayoritaria dictaminó la transgresión de los derechos de la primera enmienda de tres corporaciones closely held, en razón de la imposición del mandato contraceptivo, obligación impuesta en el Obamacare. Este artículo explica el contexto del caso, con especial mención a los fundamentos de la figura de la corporación en Estados Unidos, el reconocimiento de los derechos fundamentales para personas jurídicas, la posibilidad o no de ejercer la libertad religiosa y las particularidades del sistema de seguridad social estadounidense. Recoge los principales argumentos y hechos del caso, así como el análisis de sus implicaciones.

\section{Palabras clave}

Personalidad jurídica; libertad religiosa; Ley de Protección al Paciente y Cuidado de Salud Asequible; Obamacare; Ley de Restauración de la Libertad Religiosa. 


\begin{abstract}
The corporations as right-holders of freedoom of religion was the main topic discussed in the emblematic case of the Supreme Court of the United States Burwell $v$. Hobby Lobby. In a tight ruling five to four votes, the majority opinion founds the transgression of the I Amendment rights to three closely held corporations due to the imposition of the contraceptive mandate contained in Obamacare. This article explains the context of the case delving into the evolution of the figure of the corporation in the United States, the recognition of the fundamental rights for legal entities and the peculiarities of the American healthcare system. Also summarizes the main arguments and facts of the case and the analysis of its implications.
\end{abstract}

\title{
Keywords
}

Corporate personhood; freedom of religion; Patient Protection and Affordable Care Act; Obamacare; Religious Freedom Restoration Act. 


\section{SUMARIO}

INTRODUCCIÓN. I. ANTECEDENTES REMOTOS DEL CASO HOBBY LOBBY: 1. LOS derechos fundamentales de las corporaciones en Estados Unidos: 1.1. Algunas consideraciones sobre la figura de la corporación en el sistema legal norteamericano. 1.2. Concepto de corporación. 1.3. La figura de la corporación en la historia de Estados Unidos. 1.4. La incertidumbre sobre la titularidad de derechos fundamentales por parte de corporaciones. 2. La regulación de la seguridad social en Estados Unidos: 2.1. La Ley de Cuidado de Salud Asequible. 2.2. La Ley de Cuidado de Salud Asequible, el mandato contraceptivo y la libertad religiosa. 2.3. Las demandas judiciales a raíz de la promulgación de la Ley de Protección al Paciente y Cuidado de Salud Asequible. II. LA TITULARIDAD DE LA LIBERTAD RELIGIOSA Y EL CASO BURWELL V. HOBBY LOBBY SOTORES, INC: 1. El fallo judicial: 1.1. Los alegatos judiciales de las corporaciones con ánimo de lucro. 1.2. Los alegatos del Departamento de Salud y Servicios Sociales. 1.3. La opinión de la Corte Suprema sobre la titularidad de libertad religiosa. 1.4. La opinión desidente. 2. Las implicaciones de Hobby Lobby con respecto a la titularidad de libertad religiosa: 2.1. El reconocimiento de derechos fundamentales a una corporación closely held. 2.2. Las corporaciones con ánimo de lucro y la libertad religiosa. 2.3. El rol del Estado ante la libertad religiosa y las corporaciones. BIBLIOGRAFíA.

\section{INTRODUCCIÓN}

El presente artículo aborda la titularidad del derecho de libertad religiosa por parte de corporaciones con ánimo de lucro en Estados Unidos. Su análisis se realiza en razón del fallo Burwell v. Hobby Lobby (2014) de la Corte Suprema de Justicia de este país. El caso discute la constitucionalidad de la Patient Protection and Affordable Care Act (Ley de Protección al Paciente y Cuidado de Salud Asequible) — conocida popularmente como Obamacare - al imponer a los empleadores incorporar, y por ende, asumir, parte del coste económico en los planes de salud de sus trabajadores, programas de salud preventivos para niños, adultos y mujeres en etapa fértil. El programa preventivo cuyas beneficiarías fueran mujeres suponía el acceso a veintidós clases de servicios, entre ellos el acceso a píldoras abortivas y anticonceptivas. Esta última prestación, conocida como el contraceptive mandate (mandato contraceptivo), comprendía la obligación de facilitar dieciséis clases de anticonceptivos, entre ellos cuatro considerados abortivos. Está imposición fue, inmediatamente, reprobada 
por muchos empleadores que consideraban el hecho de facilitar este plan como contrario a sus valores religiosos, en especial, facilitar los servicios abortivos. El Gobierno, en atención a esta circunstancia, formuló una exención de cumplimiento $^{1}$ exclusiva para aquellas corporaciones ${ }^{2}$ sin ánimo de lucro, fines caritativos o religiosos, dejando de lado, arbitrariamente, a las corporaciones con ánimo de lucro.

Nos parece que para entender en profundidad lo que supone este caso en el sistema legal norteamericano es necesario, en primer lugar, el estudio de la jurisprudencia de la Corte Suprema sobre los derechos previamente reconocidos a las corporaciones, también nos resulta imprescindible exponer algunas de las particularidades del sistema de seguridad social norteamericano. A la vez intentaremos, sin tener como fin realizar un mero comentario de sentencia, relatar los hechos y el fallo del caso para aclarar que el reconocimiento de la libertad religiosa para las personas jurídicas, de acuerdo con el caso, está limitado a una clase concreta de corporación y, de acuerdo con los hechos del caso, se encuentra de por medio velar por el cumplimiento de una ley federal sobre libertad religiosa.

El artículo está dividido en dos apartados. El primero sitúa al lector con las particularidades de la figura de la corporación americana, describe sus principales características y recoge un breve relato de la jurisprudencia que les ha reconocido derechos fundamentales. En la segunda parte del primer apartado se exponen el sistema de seguridad social de Estados Unidos, las

1 Esta expresión en el presente documento hace referencia a las exenciones o excepciones que un Gobierno otorga por motivos religiosos. Esta figura se entiende como una autorización concedida a los creyentes religiosos, a nivel Legislativo, Ejecutivo o Judicial, de abstenerse de un acto que sea requerido por una ley civil (por ejemplo, la obligación de la escolarización de los niños hasta los dieciséis ańos), o permitir un acto que sea prohibido por una ley (como utilizar sustancias alucinógenas para una ceremonia), con el fundamento de que su religión lo prohíbe, lo requiere, o lo motiva a actuar o abstener de actuar de manera particular (Loewentheil, 2014; Kutoroff, 2015).

2 Está claro que no debe confundir el lector este concepto con el término «corporación» contemplado en el art. 35 del Código Civil español. Marín aclara que «las "corporaciones" a que se refiere el art. 35.1 son las modernamente denominadas, en el ámbito de la Administración estatal, organismos públicos —arts. 41 y ss. LOFAGE— [...]. También son "corporaciones", a los efectos del precepto que se comenta, los colegios profesionales (Ley 2/1974, de 13 de febrero, y normativa autonómica) y las cámaras oficiales de comercio, industria y navegación (Ley 3/1993, de 22 de marzo, y normativa autonómica)» (Marín, 2009: 151). Por ello, estas corporaciones tienen un interés público aunque la mayor parte de su actividad sea privada (De Salas, 2011: 296). 
implicaciones de la reforma al sistema llevada a cabo por el Obamacare y los conflictos de intereses de esta ley con la normativa sobre la libertad religiosa. El segundo apartado presenta los hechos del caso Hobby Lobby, los argumentos de las partes y la decisión final de la Corte. Y, por último, analiza los principales temas que sugiere la sentencia y sus implicaciones para la comprensión de una teoría sobre la titularidad de derechos.

\section{ANTECEDENTES REMOTOS DEL CASO HOBBY LOBBY}

En el 2014 la Corte Suprema de Estados Unidos emitió el fallo Burwell vs. Hobby Lobby, un caso particular al ser el demandante una persona jurídica con ánimo de lucro, un actor poco convencional para alegar libertad religiosa. En esta histórica sentencia confluyen tres cuestiones de especial relevancia en Estados Unidos: la titularidad de derechos por parte de corporaciones, la libertad religiosa y las particularidades de un sistema de seguridad social históricamente desvinculado del Estado. Este apartado está compuesto por dos partes. La primera parte busca esbozar un panorama de la titularidad de derechos fundamentales por parte de personas jurídicas en Estados Unidos, haciendo un breve recorrido tanto por la jurisprudencia como por la historia; la segunda parte relata las características del sistema de seguridad social en Estados Unidos, la novedad que supuso la adopción del Obamacare y sus repercusiones para la libertad religiosa.

\section{LOS DERECHOS FUNDAMENTALES DE LAS CORPORACIONES EN ESTADOS UNIDOS}

\subsection{Algunas consideraciones sobre la figura de la corporación en el sistema legal norteamericano}

Las nociones de persona jurídica en el sistema romano-germánico ${ }^{3}$ y en el sistema anglosajón son equivalentes. En este último la persona jurídica, de acuerdo con Black, es «un cuerpo, distinto de una persona física, que puede funcionar legalmente, demandar o ser demandado, y tomar

\footnotetext{
3 Se entiende como persona jurídica «[...] las realidades sociales a las que el ordenamiento jurídico reconoce o atribuye individualidad propia, distinta de sus miembros componentes, y a la que se trata como sujetos de derechos y deberes, con una capacidad de obrar en el tráfico por medio de sus órganos o representantes» (Díez-Picazo y Gullón, 2012: 504).
} 
decisiones a través de sus representantes legales» (Black, 2011: 419). Una persona ficticia es «una entidad, tal como una corporación, creada por la ley y con ciertos derechos y obligaciones similares a los reconocidos a una persona física; un ser real o ficticio, quien para los efectos del razonamiento jurídico se considera más o menos como un ser humano» (Black, 2011: 534).

El sistema anglosajón utiliza el término «entidad» para diferenciarlo de una persona física; esta precisión, según Villegas, "probablemente no pretende resaltar que estamos ante algo esencialmente diferente a la persona física; sino sencillamente ante un sujeto de derecho y obligaciones distinto» (Villegas, 2016: 43). En esencia supone una entidad a la que el derecho reconoce una existencia independiente y distinta a la de las personas físicas que la constituyen; esto permite que pueda ser sujeto de derechos y obligaciones y, a la vez, responsable de sus actos.

Pese a la similitud del concepto de "persona jurídica» en ambos sistemas, las formas en las que estas se pueden materializar son conceptual y terminológicamente distintas. Por ejemplo, el derecho anglonorteamericano no conoce la división entre sociedades civiles y sociedades mercantiles como sí lo hace el sistema romano-germánico. Asimismo, por seguir una tradición jurídica de Common Law, no existe una división entre el derecho público y el derecho privado; como expone Reyes, una consecuencia de esto es que «[...] una compañía puede constituirse con aportes privados o estatales, sin que dicha circunstancia implique la aplicación de una u otra rama del derecho» (Reyes, 2006: 38).

Otro elemento distintivo es la potestad que tiene cada estado de regular la creación de toda clase de personas jurídicas (Romano, 1987). Las normas que regulan la constitución, funcionamiento y estatus legal de las personas jurídicas son creaciones del derecho de cada estado, independientemente de su tamaño o de la forma en que estén distribuidas sus acciones (Morrison, 2008: 332). Lo anterior responde al hecho de que la Constitución no señala entre las materias de competencia federal la reglamentación de las sociedades (Villegas, 2016: 43). Por tanto, como afirma Vargas, «no hay en Estados Unidos un derecho de las sociedades anónimas sino tantos derechos sobre esta materia como estados» (Vargas, 1964: 25); y lo mismo cabe decir en general para todo tipo de asociaciones y sociedades.

Más allá de las disparidades en cuanto a la materialización de las sociedades entre los diferentes sistemas, a efectos del presente artículo, interesa concebir la figura de la corporación americana como una entidad con personalidad jurídica propia; con la capacidad de adquirir bienes, ejercer derechos y contraer obligaciones, y comparecer en un juicio como demandante o como demandado. 


\subsection{Concepto de corporación}

Una corporación es una ficción legal que reúne un grupo de accionistas bajo una misma personalidad jurídica, que es distinta a la de las personas físicas que la conforman, con una existencia indefinida y con los poderes derivados de su acta de constitución (Black, 2011:168). A efectos legales, las corporaciones poseen los mismos poderes que una persona natural para realizar todas aquellas labores necesarias en función de sus asuntos y negocios, es por ello que cuentan con personalidad jurídica propia. Esta condición facilita: la continuidad de contratos y la participación de activos, así como la independencia de ejercicio de los consejos de administración (Blair, 2013: 796). La corporación es la persona jurídica más utilizada, como forma organizativa de personas, en Estados Unidos debido a su independencia de las personas físicas que la conforman (Morrison, 2008: 332).

La diferencia más importante en cuanto a la clase de corporación la determina la existencia o no de lucro, y la disponibilidad de sus acciones, estas características vendrían a determinar el régimen tributario al que están sujetos. El fin de lucro es el motivo más recurrente para constituir una corporación aunque no es el único ${ }^{4}$; los accionistas pueden unirse para desarrollar actividades sociales, benéficas, religiosas, etc. La disponibilidad de las acciones también supone una división importante; existen acciones abiertas al público o cerradas. Las denominadas public corporation se denominan «abiertas» debido a que emiten acciones que pueden ser adquiridas por cualquier interesado, incluso son negociadas activamente en las bolsas de valores. Por el contrario, en las corporaciones constituidas como closely held — de especial interés para el presente artículo-, la propiedad, dirección y administración recaen en un número controlado y pequeño de accionistas, comúnmente un grupo familiar (Nicholson, 1992).

\subsection{La figura de la corporación en la historia de Estados Unidos}

La corporación se inició como una forma de agrupar personas con intereses comunes, sin embargo, con los procesos de industrialización y globalización se consolidó como unas de las principales herramientas para facilitar las transacciones comerciales; autores como Cox le otorgan un papel decisivo para el desarrollo de la industria y el comercio en los siglos xIx y xx (Cox,

4 La primera corporación en constituirse en Estados Unidos fue la Universidad de Harvard, esto manifiesta la flexibilidad de fines que permite esta figura asociativa (Brook, 1991: 140). 
2003: 2). Antes de esta época ${ }^{5}$ los grandes negocios eran administrados por un solo dueño o una sola familia (Mark, 1987: 1444). Las corporaciones eran, primordialmente, un medio para la prestación de servicios públicos ${ }^{6}$. Las primeras en recibir ese privilegio fueron las empresas dedicadas a los seguros, los bancos y los puertos, y que a su vez contaban con un capital mixto constituido con fondos públicos y privados. Después de la guerra civil, y bajo el tenor de la Revolución industrial, se hizo patente la necesidad de dirigir un negocio de manera impropia y con responsabilidad limitada. Estas características hicieron atractiva la forma de la corporación para las empresas en crecimiento, en especial para las ferrocarrileras (McBride, 2011: 3). Por tanto, a finales del XIX, la regulación de las corporaciones pasó de ser una forma organizativa con fines estrictamente estatales a una entidad creada para facilitar los negocios.

La evolución de los fines de la corporación supuso el inicio de la discusión sobre su naturaleza jurídica. La doctrina las ha agrupado en tres grupos: la teoría de la entidad artificial o por concesión; la teoría de la entidad agregada o en conjunto, y la teoría sobre la entidad real o natural (Dewey, 1926). La teoría sobre la entidad artificial se desarrolló en el xIX, cuando las corporaciones se creaban con el fin de permitir que recursos privados pudieran cumplir fines públicos. La personalidad jurídica era determinada por el Estado, que debía aprobar sus estatutos, especialmente, en los aspectos relacionados con la duración máxima, capitalización mínima, y la exigencia de dedicación a un propósito único (Marpe, 20 15: 523).

La segunda teoría se denomina teoría de la entidad agregada o del conjunto, aquí se entiende la corporación como una unión voluntaria de talentos y recursos de varios individuos, y que, por ende, los derechos de la corporación derivan de sus accionistas (Gaughan, 2012: 12). Surgió al margen de la Revolución industrial, se promovió la transición de un control total por parte del Estado a una total flexibilización de los requisitos para crear una corporación. Esto conllevó el surgimiento de actores que hasta el momento se encontraban en un segundo plano: los accionistas. (Mark, 1987: 1444; Wood y Scharffs, 2002: 542).

5 «En todo el período colonial, solo siete empresas se constituyeron en las colonias británicas de Norteamérica. En tan solo los últimos cuatro ańos del siglo dieciocho, sin embargo, se constituyeron trescientas treinta y cinco empresas en los nuevos Estados Unidos» (McBride, 2011: 3).

6 Nace expone una tabla con los controles que existían para las corporaciones antes de la guerra civil. Algunos de estos son: la limitación por parte de los estatutos a la cantidad de dinero que una corporación podía ganar; generalmente poseían un plazo de vigencia de veinte a cincuenta ańos, y ninguna corporación podía realizar actividades en otro Estado (Nace, 2003: 51-52). 
Por último, la teoría sobre la entidad real o natural considera la corporación como una persona jurídica independiente de sus accionistas. Esta nueva persona jurídica puede incluso gozar de los mismos derechos que las personas físicas pero no por el hecho de derivar de sus accionistas. Se considera que el emblemático caso Santa Clara County v. Southern Pacific Railroad Corporation (1886) dio inicio a esta teoría al considerar a la corporación como una "persona».

Las tres teorías, propuestas por la doctrina, intentan buscar parámetros dentro de la jurisprudencia; sin embargo, la Corte Suprema no ha reconocido ninguna de ellas; autores como Dewey consideran que la discusión de estas teoría es un debate que no aporta claridad para la definición sobre la naturaleza de las personas jurídicas (Dewey, 1926). Krannich aporta más precisión al debate al proponer un análisis de titularidad en razón de la naturaleza del derecho $^{7}$, esto permite que se les reconozcan solo aquellos derechos derivados de su personalidad jurídica (Krannich, 2005: 108). A continuación se expone cuál ha sido la construcción legal para el reconocimiento de derechos fundamentales de las corporaciones.

\subsection{La incertidumbre sobre la titularidad de derechos fundamentales por parte de corporaciones ${ }^{8}$}

El sistema legal de Estados Unidos carece de una teoría definida sobre la naturaleza de la persona jurídica. Hovenkamp ha catalogado este debate como «uno de los mayores malentendidos de la doctrina en la historia legal de

7 En países como España y Alemania el tema parece ser menos controvertido. En Espańa son numerosas las sentencias del Tribunal Constitucional que abordan la materia, desde la Sentencia 139/1995 se reconoce que los derechos fundamentales rigen también a las personas jurídicas nacionales en la medida en que por su naturaleza le resulten aplicables. Gómez Montoro, en referencia a la situación de España, utiliza una expresión muy parecida a la de Krannich (2005) «es necesario determinar caso por caso qué derechos fundamentales pueden reconocerse a cada entidad organizativa [...]» (Gómez Montoro, 2002: 104). En Alemania la jurisprudencia, en torno al análisis de los arts. 19.3 GG y 1 de la Ley Fundamental, apunta al reconocimiento de los derechos fundamentales de las personas jurídicas con motivo de la dignidad de las personas que conforman dicha ficción legal (Gómez Montoro, 2002: 80-81 y 98-99).

8 El derecho constitucional norteamericano, al momento de reconocer algún derecho fundamental, se centra en una primera etapa, en la existencia de standing, esto es, el interés legítimo para recurrir. Una vez que se acredite este interés, los fallos analizan la titularidad del derecho en cuestión por parte del recurrente de acuerdo con los hechos del caso. 
Estados Unidos» (Hovenkamp, 1988: 1649). En este sistema jurídico la titularidad de los derechos fundamentales depende del lenguaje utilizado en la Constitución. El texto constitucional dispone el disfrute de algunos derechos de acuerdo con la condición de "persona» o de «ciudadano» (Krannich, 2005: 61), aunque no existe una postura clara sobre si bajo "persona» se entiende incluidas a «las personas jurídicas», situación que no es muy clara debido a que las expresiones "persona jurídica» o "corporación» están ausentes en el texto constitucional?.

El Code of law of the United States es el instrumento jurídico que aporta un poco de luz al debate de la naturaleza de las corporaciones. En esta compilación de leyes federales se indica, en su primer apartado, la igualdad de trato para las corporaciones y las personas físicas, a menos de que el contexto indique lo contrario (Wood y Scharffs, 2002: 553). La afirmación «a menos de que el contexto indique lo contrario" parece indicar una serie de derechos que la persona jurídica no podría ejercer por su naturaleza «no humana», por ejemplo: el matrimonio; además de aquellos que se entienden reservados exclusivamente para «los ciudadanos» — concepto que normalmente excluye a extranjeros y a personas ficticias - (Eberle, 2003: 23). En general, se admite que las corporaciones gozan de una serie de derechos que han sido reconocidos por la jurisprudencia. Desde 1809 la Corte Suprema reconoció que las corporaciones tenían derechos fundamentales ${ }^{10}$. Sin embargo, debido a las características antiteóricas y pragmáticas del sistema norteamericano ante el abordaje de cuestiones como los derechos fundamentales, el desarrollo jurisprudencial de este tema ofrece un panorama heterogéneo e incluso contradictorio ${ }^{11}$.

En Trustees of Dartmouth College v. Woodward (1819) la Corte establece que los estatutos de la corporación son un contrato y el intento de modificarlos

9 La escaza existencia de corporaciones al momento de redactarse la Constitución Política de Estados Unidos es uno de los motivos históricos por los que no se les menciona en dicho texto (Pollman, 2011: 1633).

10 Bank of the United States v. Deveaux, 9 (5 Cranch) U.S. 61.

11 Visiones opuestas se pueden observar en dos casos de 1978. En el primero, First National Bank v. Bellotti, considera inaccesibles para las corporaciones los derechos fundamentales debido a que por razones de finalidad, historia y naturaleza pertenecen exclusivamente a las personas físicas. En el segundo caso, Monell v. Department of Social Service, dictaminó que desde 1871 — con la promulgación del Civil Rights Act of 1871 , instrumento que supuso el resguardo de los derechos de la población afroamericana- se entendía que las corporaciones estaban incluidas dentro del término "persona» salvo cuando el contexto indicara lo contrario (Wood y Scharffs, 2002: 548). 
por parte del Estado es una violación a su libertad contractual, reconocida en el art. 1, sección 10. ${ }^{\text {a }}$ Este fallo es considerado como la génesis de la actual estructura legal de las corporaciones. Por su parte, la cláusula de igual protección se les reconoce a las corporaciones en Santa Clara County v. Southern Pacific Railroad Co. (1886), al otorgarles la condición de "personas» para los efectos de gozar de la XIV enmienda ${ }^{12}$.

La libertad de expresión se les reconoce a las personas jurídicas en First National Bank of Boston v. Bellotti (1978). Algunas corporaciones, para manifestar su oposición a la adopción de un impuesto progresivo, destinaron ciertas cantidades de dinero a un determinado partido político que defendería, en un fututo, mejor sus intereses. El estado de Massachusetts prohibió a las corporaciones brindar esta clase de apoyo, sin embargo, la Corte Suprema consideró esta imposición como inconstitucional. Este fallo ha sido citado posteriormente en el polémico caso Citizens United v. Federal Election Commission (2010), donde se prohibió al Gobierno restringir un tope de gastos en política a una corporación sin ánimo de lucro.

En New York Times v. Sullivan (1964) considera a las corporaciones garantes de los derechos recogidos en las enmiendas I y XIV. La Corte valora la importancia de salvaguardar la libertad de expresión de las empresas dedicadas a los medios de comunicación masivos. En cuanto a la libertad de expresión como estrategia comercial, en Virginia State Board of Pharmacy v. Virginia Citizens Consumer Council (1976) se declara inconstitucional una ley del estado de Virginia que prohibía a los farmacéuticos anunciar los precios de sus productos. Sin embargo, el caso aborda la problemática con mayor énfasis en el derecho del consumidor de conocer el precio de los productos.

El derecho al debido proceso se discute en la sentencia Noble v. Union River Logging Railroad Co. (1893). La empresa ferrocarrilera Union River Logging Railroad gozaba del derecho de vía a través de un terreno público, sin embargo, la Administración rescinde unilateralmente la concesión al descubrir que la empresa se estaba dedicando a la tala forestal. La corporación acude a la Corte Suprema alegando el derecho al debido proceso, debido a su condición de persona, que tiene reconocido según la $\mathrm{V}$ enmienda; finalmente la empresa obtiene una decisión favorable (Nace, 2003: 232).

12 «Las corporaciones son personas bajo los términos de la Décimo Cuarta Enmienda de la Constitución de los Estados Unidos». Esta cita corresponde concretamente al encabezado del fallo y en ella se afirmaba que todos los jueces coincidían con que las corporaciones debían disfrutar de los derechos consagrados en dicha Enmienda. Sin embargo, esta fue una interpretación hecha por el taquígrafo personal del presidente de la Corte Suprema, incluso este punto es inexistente en el fallo (Magrath, 1963). 
La IV enmienda, relativa a la interdicción de registros e incautaciones irrazonables, ha sido interpretado por la Corte Suprema más como una protección de un derecho colectivo que de un derecho individual, y suele contrastarla con los intereses gubernamentales (Clancy, 2010: 263). Pese a que a las corporaciones se les reconoce este derecho desde Hale v. Henkel (1906) y establece que solo deben de cumplir aquellas citaciones judiciales que sean razonables, en United States v. Morton Salt Co. (1950), la Corte Suprema estableció que las corporaciones no pueden reclamar igualdad de derechos en relación con las personas en cuanto a la protección de la IV enmienda. La doctrina sostiene que este criterio se debe a la importancia de las inspecciones administrativas para velar por el cumplimiento de la ley en los negocios (Garrett, 2014: 124).

En Hale v. Henkel (1906) también se analiza el derecho a no autoincriminarse reconocido en la $\mathrm{V}$ enmienda. El fallo cataloga el derecho a no testificar como un beneficio exclusivo de las personas físicas en atención al respeto de su vida privada; una persona jurídica, siendo una creación del Estado, no tiene el derecho de negarse a presentar sus libros y papeles ante una autoridad alegando una supuesta privacidad. El derecho de no ser sometidos a una doble incriminación fue discutido por primera vez en United States v. Armco Steel Corporation (1966); la Corte establece que dicho privilegio, otorgado en fallos posteriores a las personas, debe extenderse a las corporaciones, ya que estas también son "personas" de acuerdo con el Code of law of the United States. Analizando el mismo derecho en United States v. Security National Bank (1976), la Corte señala los efectos económicos, la incertidumbre e inseguridad que generaría en una persona jurídica, independientemente de su tamaño, el hecho de tener que afrontar un proceso legal dos veces (Kane, 1977).

El derecho a un juicio con jurado en materia penal y civil, consignado en las VI y VII enmiendas, se ha reconocido a las personas jurídicas en razón de habérseles garantizado el derecho al debido proceso. En el fallo Southern Union v. United States (2012), la Corte Suprema consideró como una violación a los derechos de la VI enmienda imponer una multa por contaminación ambiental a una empresa basada en hechos que no habían sido conocidos por el jurado; sin embargo, este caso se resolvió por un aspecto procesal del jurado y es débil el análisis de la titularidad del derecho por parte de las personas jurídicas.

La anterior recopilación no pretender ser exhaustiva, pero apuesta por presentar al lector un panorama sobre los derechos fundamentales reconocidos a las corporaciones por la jurisprudencia hasta el día de hoy. Queda patente la ausencia de una teoría general sobre la naturaleza o la titularidad de derechos por parte de las corporaciones. En cada caso el razonamiento dado por la Corte está sujeto a la forma corporativa de la que se trate y la clase de derecho que se alegue. 
Desde el año 2010, con el fallo Citizens United v. Federal Election Commission, y continuando en 2014 con el caso Burwell v. Hobby Lobby, la titularidad de derechos fundamentales por parte de las personas jurídicas ha generado una especie de "nerviosismo social» tanto en la doctrina como en la opinión pública, al considerar que los fallos, progresivamente, buscan la equiparación de los derechos de las personas físicas con los de las corporaciones, incluso para derechos puramente personales - matrimonio, voto, etc.- . Algunos sectores de la doctrina no cesan de catalogar los fallos que reconocen algún derecho fundamental a las corporaciones como «arbitrarios», «falaces» o «desproporcionados» (Krannich, 2005; Mayer, 1989; O’Kelley, 1979).

En reacción a estos dos fallos, diversos grupos reclaman un control estricto o una total eliminación de los derechos fundamentales para las corporaciones ${ }^{13}$. La filosofía, la psicología e incluso la sociología han tomado parte en esta interminable discusión (Ripken, 2011; Brown, 2013). La opinión pública, por su parte, utiliza caricaturas de corporaciones como «monstruos» (McFadden, 2016), «profesores» (Bulling, 2011) e incluso como beneficiarios de derechos procesales superiores a los de los seres humanos (Rall, 2016). Algunos sectores de la doctrina consideran el reconocimiento de derechos fundamentales para las corporaciones como desproporcionales. Autores como Mayer, en referencia a las peticiones judiciales hechas por las corporaciones de las diez primeras enmiendas, pronostica la transición de un sistema judicial tradicional en el que se vela por las libertades individuales a un sistema de justicia constitucional centrado en atender exclusivamente peticiones de organizaciones (Mayer, 1989: 578).

13 Diferentes estados, como Colorado y Montana, han promovido proyectos para enmendar la Constitución con el fin especificar que las corporaciones no son personas jurídicas ( State Initiatives to Revoke Corporate Personhood and Overturn Buckley v. Valeo Win Big», 2012). Organizaciones como Move to Amend y Alliance for Democracy han organizado sitios webs en contra de la corporate personhood, por ejemplo: movetoamend.org y www.thealliancefordemocracy.org/corprule.html. A nivel federal, el congresista Ted Deutch, por Florida, y el Senador Bernie Sanders han promovido el documento S. J. Res. 33, 112 Cong. (2011), en el que se propone una enmienda constitucional para excluir expresamente a las corporaciones con ánimo de lucro de los derechos otorgados a personas físicas por la Constitución de Estados Unidos. Otra iniciativa de interés es el documento H.R.J. Res. 88, 112 Cong. (2011), donde se sugiere una enmienda constitucional que indica que «al decir "gente", "persona" o "ciudadano" tal como se utiliza en esta Constitución no se incluyen corporaciones, sociedades de responsabilidad limitada u otras entidades corporativas» (US Government Publishing Office - FDsys - Home, s. f.). 


\section{LA REGULACIÓN DE LA SEGURIDAD SOCIAL EN ESTADOS UNIDOS}

La Constitución de Estados Unidos no reconoce la existencia de un derecho a la salud ni prevé un sistema de seguridad social público y nacional. Sin embargo, el goce de algunos beneficios sociales se ha construido de acuerdo con el art. I, sección VII del texto constitucional; esta disposición reconoce el poder que tiene el Gobierno federal, concretamente el Congreso, para aumentar los impuestos a los servicios de salud y velar por el bienestar general de la población. De esta forma el Gobierno asigna fondos a los estados con el fin de llevar a cabo actividades para el bienestar general de la población, así como la potestad de fijar políticas para la utilización de los fondos asignados.

De acuerdo con el último censo, un $90,9 \%{ }^{14}$ de la población de Estados Unidos cuenta con un seguro médico y un 67,2\% de esos beneficiarios utiliza un seguro privado ${ }^{15}$. La intervención de un tercero - ya sea el empleador o el Gobierno-, que efectúa el pago a los proveedores de los seguros médicos, es lo que faculta a un ciudadano para acceder a los servicios de salud. La autoridad administrativa en temas de salud recae en el Department of Health and Human Services (Departamento de Salud y Servicios Sociales), que vela por la regulación de los seguros médicos y dirige los servicios sociales esenciales que se brindan a grupos de beneficiarios concretos: militares, etc.

\subsection{La Ley de Cuidado de Salud Asequible}

El sistema de seguridad social norteamericano es el producto de múltiples reformas de acuerdo con los intereses de cada momento histórico (Kollmann, 1996). En la actualidad la optimización del servicio tiene dos objetivos concretos: la disminución del coste económico de la asistencia sanitaria y la reducción del porcentaje de personas sin seguro médico. Con el fin de

14 En 2015, el porcentaje de personas sin cobertura de seguro de salud durante todo el año calendario fue de 9,1\% o 29 millones de personas. Está cifra es inferior a la tasa y el número de personas sin seguro en 2014, que correspondió a un 10,4\% o 33 millones (Barnett y Vornovitsky, 2015).

15 En un contexto europeo estas cifras pueden parecer alarmantes. Los sistemas europeos desde un inicio se caracterizaron por ofrecer sanidad desde la perspectiva de un Estado de bienestar. Alemania adoptó en 1883 un seguro de salud para los trabajadores y seis años después instituyó un sistema nacional de pensiones. Inglaterra creó un seguro de salud por invalidez en 1911 y en 1925 incluyó un seguro por vejez. Por su parte, Estados Unidos decretó su primera ley nacional de seguros en 1935, cuando más de veinte países en todo el mundo contaban con sistemas de seguridad social (DeWitt, 2010: 3). 
acometer estas metas se promulgó, el 23 de marzo de 2010, el Obamacare. Con más de novecientas páginas, cientos de reformas y la expectativa de ser implementado a lo largo de varios años, el Obamacare supuso una ambiciosa reforma a todo el sistema de salud (United States. Department of Health and Human Services, 2013). E incluso puede considerarse como el primer intento del sistema norteamericano de otorgar el acceso a la salud como un derecho fundamental a todos los ciudadanos.

\subsection{La Ley de Cuidado de Salud Asequible, el mandato contraceptivo y la libertad religiosa ${ }^{16}$}

Durante la etapa de elaboración del proyecto de ley, uno de los temas centrales fue la diferencia entre el coste económico y los distintos servicios que deben costearse hombres y mujeres. Se entendía que, debido a la maternidad, las mujeres deben asumir un gasto mayor para cubrir diversos servicios médicos y medicamentos relacionados, de una u otra forma, con los cuidados prenatales y posnatales; así como las medidas anticonceptivas y abortivas. Se estableció que una mujer, durante su etapa fértil, realizaba una inversión un $68 \%$ más alta que un hombre (Benson, 1998). Algunos senadores, en especial Bárbara A. Mikulski y Dianne Feinstein, se propusieron lograr reducir esta brecha recargando en los empleadores parte del costo que estaban asumiendo las mujeres, y proyectando su beneficio indirecto para cónyuges, hijas, etc. (Feinstein, 2009).

El Consejo delegó en el Departamento de Salud y Servicios Sociales la elaboración de un informe con las recomendaciones sobre aquellos servicios preventivos indispensables para las mujeres en etapa fértil. En el informe se definieron los servicios preventivos como medidas «que se contemplan para mejorar el bienestar, y/o disminuir la probabilidad de aparición de una enfermedad o condición específica» (Institute of Medicine of the National Academies, 2011). Por tanto, recomendó introducir en los planes de seguros médicos para mujeres, en etapa fértil: exámenes preventivos — servicios prenatales,

16 En el momento en el que se concluye este artículo el presidente electo Donald Trump ha prometido en su plan de gobierno eliminar el Obamacare y reemplazarlo por un plan de salud acorde con los principios del libre comercio («Donald J. Trump for President», s. f.). En el primer día en su cargo, el presidente Trump ha emitido una orden ejecutiva indicando una sustitución progresiva del Obamacare («Executive Order Minimizing the Economic Burden of the Patient Protection and Affordable Care Act Pending Repeal», 2017). Los senadores republicanos Susan Collins y Bill Cassidy presentaron, tres días después de la orden ejecutiva, el Patient Freedom Act como una opción sustitutiva. 
mamografías...-, exámenes de detección — diabetes gestacional, VIH, VPH...-, asesoría profesional para afrontar la violencia doméstica y para una correcta lactancia, además de todos los métodos anticonceptivos aprobados por la Food and Drug Administration (Administración de Medicamentos y Alimentos). El informe fue acogido completamente y oficializado el 1 de agosto de 2011.

La obligación de proveer anticonceptivos en los planes de salud se popularizó como el mandato contraceptivo. Desde un inicio dicha obligación fue rechazada por los grupos cuyos valores son contrarios a los métodos de control de la natalidad —organizaciones religiosas, corporaciones sin ánimo de lucro, etc.- . En respuesta a esta situación el Gobierno procedió a conceder una exención por motivos religiosos ${ }^{17}$ exclusivamente para los beneficiarios cuyo proveedor fuera un «empleador religioso».

Para definir «empleador religioso» se establecieron cuatro criterios indispensables: 1) un empleador que tiene como fin primordial infundir valores religiosos; 2) sus empleados son, primordialmente, personas que comparten sus principios religiosos; 3) los servicios que brinda están dirigidos, especialmente, a personas que comparten sus principios religiosos; y 4 ) que está constituida como una organización sin ánimo de lucro de acuerdo con la sección 6033 a.1 y la a.3.A.i del Código Fiscal de Estados Unidos; apartado que se refiere principalmente a las iglesias y sus instituciones asociadas («Coverage of certain preventive services under the Affordable Care Act», 2013).

Durante la elaboración del mandato contraceptivo se presentaron más de doscientos mil comentarios de la sociedad civil acerca del concepto de «empleador religioso». Las manifestaciones eran radicalmente opuestas: un grupo solicitaba ampliar el concepto para incluir más empleadores con una afinidad religiosa, y el grupo contrario abogaba por mantener la definición, ya que de lo contrario se limitaría el acceso a los métodos anticonceptivos. Pese a la

$17 \mathrm{Al}$ ser un caso en el que se discute la libertad religiosa, el Gobierno debe cumplir lo establecido en la Ley de Restauración de Libertad Religiosa (Religious Freedom Restoration Act), cuyo fin principal es el de declarar la invalidez de una ley si esta impone una carga significativa al ejercicio de la religión de una persona. Esta ley, en su apartado b, menciona las condiciones que deben cumplirse para justificar una vulneración de la libertad religiosa: 1) la existencia de un fin público imperioso compelling interest_- y 2) utilizar el medio menos restrictivo para conseguir dicho fin. $\mathrm{Al}$ crear el concepto de «empleador religioso", el Gobierno pretende cumplir con el punto 2 de la mencionada ley federal, por ende, prevé una vía para que todas aquellas personas que se puedan ver afectadas por sus creencias religiosas se vean exentas de cumplir dicha ley o su vulneración sea la mínima posible. 
multitud de alegatos y protestas, la Administración mantuvo la definición. A la vez concedió el plazo de un ańo para que las corporaciones, sin ánimo de lucro y con una misión religiosa, pudieran solicitar, mediante una gestión administrativa, abstenerse del mandato contraceptivo.

Los cuatro criterios indispensables para ser considerado «empleador religioso» no consideraban las diversas relaciones comerciales y laborales que realizan escuelas, universidades, hospitales y organizaciones benéficas con idearios religiosos. Algunas de las situaciones que les impedían cumplir con dichos criterios fueron el hecho de brindar servicios a individuos que no compartían sus mismas creencias; o su que finalidad era distinta a la de inculcar valores religiosos; o que empleaban un número de personas que no compartían sus creencias religiosas, o tenían ánimo de lucro.

Ante estas situaciones, el 1 de febrero de 2013, el Gobierno manifestó que ampliaría el concepto de "empleador religioso" para incluir a todas aquellas organizaciones que pudieran verse afectadas por su ideario religioso. Por tanto, emitió una disposición final, estableciendo unos requisitos, más flexibles, para acogerse a la exención. Las nuevas disposiciones abarcaban a las organizaciones que 1) se negaran a proporcionar cobertura para alguno o todos los servicios de anticoncepción por convicciones religiosas, 2) eran corporaciones sin ánimo de lucro, 3) se autodefinían como una organización religiosa, y 4) contaran con un documento que diera fe de que cumplían los tres criterios anteriores. Este cambio hizo posible que instituciones religiosas constituidas como corporaciones sin ánimo de lucro - organizaciones benéficas, educativas y otras organizaciones religiosaspudieran abstenerse del mandato contraceptivo. Sin embargo, expresamente se estableció que las corporaciones con ánimo de lucro no podrían optar por esta excepción («Coverage of certain preventive services under the Affordable Care Act», 2013).

Los «empleadores religiosos» no fueron los únicos exentos de cumplir el mandato contraceptivo. Los empleadores con grandfathered plans — planes constituidos antes del 23 de marzo de 2010 - y las empresas pequeñas aquellas con una cantidad de trabajadores inferior a cincuenta- quedaron exentos. Las organizaciones fuera de las anteriores excepciones estaban obligadas a cumplir con el mandato contraceptivo o de lo contrario podrían ser sometidas al pago de elevadas sanciones económicas.

\subsection{Las demandas judiciales a raíz de la promulgación de la Ley de Protección al Paciente y Cuidado de Salud Asequible}

El Obamacare impuso una serie de obligaciones en un sistema jurídico marcadamente liberal, por lo que las demandas judiciales alegando su 
inconstitucionalidad surgieron inmediatamente después de su publicación. Sin duda el apartado del Obamacare más sometido a litigios fue el mandato contraceptivo, en especial por su alegado contenido moral, llegando a contabilizar más de cien demandas judiciales (National Women's Law Center, 2015). Las apelaciones se presentaron incluso tres meses antes de su publicación, como fue el caso de la universidad católica Belmont Abbey College, e incluso la empresa Eternal Word Television Network presentó su demanda un día después de publicada la ley.

Al debate moral y judicial promovido por las corporaciones sin ánimo de lucro se le unieron varias demandas judiciales de aquellas constituidas con ánimo de lucro; como se señaló anteriormente, estas habían quedado fuera de la exención otorgada por el Gobierno. Cuatro circuitos judiciales ${ }^{18}$ entraron a conocer esta cuestión; tres de ellos consideraron el ejercicio de la libertad religiosa incompatible con la persona jurídica. El Sexto y el Tercer circuito fallaron en contra alegando la existencia de derechos «puramente personales» $\mathrm{y}$, por ende, inaccesibles para las corporaciones. A la vez, consideraron la naturaleza de las creencias religiosas como inherentemente humanas y recalcaron la inexistencia de jurisprudencia sobre el asunto (United States Court of Appeals for the Third Circuit, 2013: 22). Por su parte, el Circuito del Distrito Federal, en Gilardi v. U.S. Department of Health \& Human Services, tomó una posición intermedia al negar a Freshway Foods corporation la posibilidad de alegar una violación de derechos pero aclaró que sus propietarios podrían plantear una demanda a título personal.

Por el contrario, el Décimo Circuito en Hobby Lobby v. Sebelius reconoció la posibilidad de la corporación de ser sujeto de estos derechos; consideró el derecho a profesar una religión como un derecho no exclusivamente personal y equiparó los derechos de las corporaciones con ánimo de lucro a los reconocidos a las sin ánimo de lucro (United States Court of Appeals for the Tenth Circuit, 2013: 7). Pese a que este circuito reconoce la titularidad de la libertad religiosa para la empresa Hobby Lobby, concluye que el mandato contraceptivo es obligatorio para esta y no para sus dueños, de modo que las obligaciones son independientes y, por ende, afectarían a personas distintas, lo que implica que la empresa debe cumplir. Las partes vencidas en los cuatro circuitos judiciales presentaron apelación ante la Corte Suprema de Justicia; la Corte unificó los argumentos y decidió escuchar los casos de Conestoga Wood Specialities y Hobby Lobby.

18 Los circuitos resuelven las apelaciones contra las decisiones de las cortes de distrito dentro de su circuito judicial federal, y en algunos casos de otros tribunales federales así designados y de las agencias u organismos administrativos. 


\section{LA TITULARIDAD DE LA LIBERTAD RELIGIOSA Y EL CASO BURWELL V. HOBBY LOBBY STORES, INC}

\section{EL FALLO JUDICIAL}

\subsection{Los alegatos judiciales de las corporaciones con ánimo de lucro}

Hobby Lobby y Conestoga no encajaban en ninguno de los presupuestos contenidos en la exención religiosa: habían sido constituidas como corporación con ánimo de lucro, no tenían un grandfathered plan, ni menos de cincuenta empleados. Ambas empresas poseen una estructura e ideario empresarial marcadamente religiosos, características por las que alegan la violación a la libertad religiosa de sus empresas de acuerdo con la I enmienda y, concretamente, bajo la Ley de Restauración de la Libertad Religiosa. Los demandantes no se oponían a la totalidad del mandato contraceptivo, sino exclusivamente a lo referente a los tratamientos considerados abortivos: dos clases de la llamada "píldora del día después» y dos tipos de dispositivos intrauterinos.

$\mathrm{Al}$ ser un supuesto donde se considera la eventual violación de la Ley de Restauración de la Libertad Religiosa, se debe demostrar a nivel judicial la existencia de unos «sinceros religiosos» por parte de la víctima; la Corte, por su parte, debe comprobar exclusivamente la presencia de estos valores y no puede manifestar su opinión sobre la veracidad de esas creencias (Gedicks, 2017). Las personas jurídicas, como parte del proceso judicial, exponen la presencia de los valores religiosos en sus empresas. Conestoga Wood Specialties es una corporación constituida como closely held — sus acciones están cerradas al público-, su patrimonio y junta directiva pertenecen exclusivamente al matrimonio Hahn y sus tres hijos, todos ellos miembros de la Iglesia menonita. Los valores religiosos de esta agrupación religiosa son contrarios al aborto y apoyan el inicio de la vida desde la concepción. Los Hahns conducen su negocio de acuerdo con sus creencias religiosas y sus principios morales; toda la junta directiva ha ratificado la «Declaración de la familia Hahn sobre la santificación de la vida humana», donde se reconoce que la vida se inicia con la concepción y se considera contrario a sus convicciones morales el verse envueltos en procedimientos que interrumpan la vida. Si la empresa actuara de acuerdo con sus convicciones y por ende no cumpliera con dicha obligación, supondría una penalización de 95000 dólares por día. El principal argumento de Conestoga reside en el hecho de que los individuos pueden ejercer la religión a través de un negocio, independientemente de la forma asociativa utilizada, además sostienen que los precedentes de la Corte Suprema no encasillan la actividad lucrativa como algo incompatible con el ejercicio de la religión (Alliance Defending Freedom, 2014: 12). 
Las otras corporaciones que forma parte del litigio son Hobby Lobby y Mardel, ambas propiedad de la familia Green, también constituidas como closely held y con unos marcados valores cristianos en la administración de ambos negocios. Hobby Lobby tiene como uno de sus propósitos «dar gloria al Señor en todo lo hecho para el manejo de la empresa de una manera coherente con los principios de la Biblia». Cada miembro de la familia firmó un compromiso para dirigir ambas empresas de acuerdo con las creencias religiosas familiares; este compromiso se materializa al realizar importantes donaciones a ministerios cristianos, cerrar sus tiendas los domingos, y contar con servicio de capillas, consejeros y cursos espirituales para sus empleados. Para Hobby Lobby, la penalización por no cumplir con el mandato contraceptivo suponía una penalización excesiva - teniendo en cuenta que más de trece mil personas estaban aseguradas bajo su plan de salud-, la empresa tendría dos opciones: pagar una multa por no cumplir con el mandato en concreto - lo que supondría, por lo menos, 1,3 millones de dólares por día y casi 475 millones por año-o abstenerse de brindar cualquier clase de seguro y pagar una multa de 26 millones de dólares al ańo (Corte Suprema de Estados Unidos, 2013b: 18). Las cifras anteriores confirman la protección reforzada del Obamacare por el mandato contraceptivo hasta el punto de ser altamente penalizado el hecho de incumplir exclusivamente con él.

\subsection{Los alegatos del Departamento de Salud y Servicios Sociales}

La Administración defiende la constitucionalidad del mandato contraceptivo a través de tres argumentos. El primero apunta al carácter impersonal que existe entre el vínculo entre los dueños de las empresas y el plan de salud que deben proveer a sus trabajadores. Esta es una obligación que no recae sobre los dueños de las corporaciones sino sobre su empresa, que, pese a ser de su propiedad, es una persona diferente; la eventual muerte de embriones la causaría una persona distinta a los dueños. Apoya este argumento en la existencia del velo societario que desvincula a la corporación de sus accionistas.

El segundo argumento expone la incapacidad de una corporación de ser titular de la libertad religiosa bajo la Ley de Restauración de la Libertad Religiosa. Las corporaciones no pueden considerarse personas ni existe algún motivo por el que el Congreso pretenda conceder a las corporaciones con ánimo de lucro derechos exclusivos para el ejercicio religioso de los individuos e instituciones religiosas sin ánimo de lucro (Corte Suprema de Estados Unidos, 2013a: 18). Otorgar una exención a una empresa que se dedica al lucro puede generar, eventualmente, prácticas abusivas y desleales.

El tercer argumento sustenta la disponibilidad de los anticonceptivos para las mujeres como una medida que favorece la igualdad de género y que, 
por ende, es de interés público. La reducción del porcentaje de madres solteras y embarazos no deseados, o los beneficios de contar con un índice de inferior natalidad, entre otros, supondrían un avance para la igualdad de género (Corte Suprema de Estados Unidos, 2013a: 15). Considera esta obligación como un programa de salud pública que debe prevaler sobre los derechos de los empleadores, además, no se pueden menoscabar los derechos de los trabajadores por la primacía de las creencias religiosas de los empleadores. Concluye, escuetamente, en el rol del empleador como un actor esencial para contar con un sistema de salud, ya que el Estado no debe afrontar un deber que corresponde a los empleadores (Corte Suprema de Estados Unidos, 2013b: 24).

\subsection{La opinión de la Corte Suprema sobre la titularidad de libertad religiosa}

El voto mayoritario, redactado por el juez Alito y apoyado por cinco de los nueve jueces, soluciona encadenadamente las siguientes cuestiones: la titularidad de las corporaciones con ánimo de lucro para alegar la violación de sus derechos de acuerdo con la Ley de Restauración de la Libertad Religiosa, y el análisis del apartado $b$ de dicha ley, que determina la legalidad de la vulneración de la libertad religiosa, esto es, la existencia de un 1) fin público y 2) la utilización del medio menos restrictivo para cumplir dicho fin.

El fallo inicia analizando la titularidad de las corporaciones de alegar sus derechos de acuerdo con la categoría de "persona» del Dictionary Act — glosario de conceptos de leyes federales-. En él se lee: «El significado de cualquier ley del Congreso, a menos que el contexto indique otra cosa, la palabra "persona" incluye corporaciones, empresas, asociaciones, firmas, sociedades, y las sociedades por acciones, así como individuos» (Corte Suprema de Estados Unidos, 2014: 19). De esta manera, la Corte faculta a las corporaciones con ánimo de lucro a ser titulares de la protección a la libertad religiosa bajo la Ley de Restauración de la Libertad Religiosa. Determina que es inconstitucional establecer una división entre las corporaciones en razón del lucro; aclara que, aunque el fin de lucro suele ser el más recurrente en estas formas organizativas, este no es el único e incluso se dedican a múltiples causas, entre ellas, las religiosas.

La Corte establece que el mandato contraceptivo vulnera la libertad religiosa de las corporaciones. En primer lugar, el interés público que intenta cumplir el Gobierno se cumple precariamente debido a dos motivos: los intereses son muy generales y es cuestionable el impacto social del proyecto, tomando en consideración que se permitió a muchos empleadores eximirse de su cumplimiento y, por ende, su ámbito de cobertura se limitó considerablemente. Además, la Administración pudo haber previsto un plan anticonceptivo que no incluyera los cuatro métodos que conllevan la muerte del embrión. 
En segundo lugar, el mandato supone una vulneración injustificable a los valores religiosos de los dueños de las corporaciones. Expone a los empleadores a severas sanciones económicas y no existe una vía alternativa para evadir su cumplimiento. Enfatiza en su incapacidad para determinar la razonabilidad o validez de las creencias religiosas de sus ciudadanos, sin embargo, manifiesta que ambas empresas han demostrado sólidas y coherentes manifestaciones religiosas en la administración de sus negocios.

\subsection{La opinión disidente}

Cuatro magistrados se apartan de la opinión mayoritaria. La magistrada Ginsburg se erige como la disidente primaria recibiendo el apoyo, al menos en parte, de tres jueces más. Niega la aplicabilidad de la Ley de Restauración de la Libertad Religiosa para las corporaciones, ya que entiende que la ley tiene un contexto y no es necesario recurrir al Dictionary Act, sostiene que la titularidad de la libertad religiosa es exclusiva para personas humanas. Agrega que las organizaciones religiosas existen para fomentar la fe de aquellas personas con las mismas creencias y, por el contrario, las corporaciones agrupan a personas de diferentes credos y sus fines distan de fomentar la fe. Manifiesta su preocupación ante la «expansión» de los derechos fundamentales para las corporaciones y la utilización de las creencias religiosas como un medio para evadir el cumplimiento de la ley. Este último argumento es compartido por la jueza Sotomayor; sin embargo, los jueces Breyer y Kagan comparten los argumentos sobre el interés público del mandato contraceptivo pero consideran que las corporaciones con ánimo de lucro pueden ser titulares de la libertad religiosa. Por ende, los jueces Breyer y Kagan se unen en este punto al voto mayoritario, lo que implica que siete de los nueve jueces — sentando un precedente importante — sostienen que las corporaciones pueden sufrir un menoscabo a su libertad religiosa y, por ende, son titulares.

En su opinión, el fallo principal es erróneo al permitir a las corporaciones oponerse a cualquier ley que juzguen incompatible con sus creencias religiosas, y sopesa el interés para las mujeres de los servicios del mandato contraceptivo. La Ley de Restauración de la Libertad Religiosa no fue promulgada para la imposición de creencias religiosas; ejemplifica su argumento con un elenco de casos donde el reconocimiento de la libertad religiosa del empleador ha supuesto una violación de los derechos fundamentales de otros ciudadanos. Algunos de estos casos son: un propietario de una cadena de restaurantes se negó a brindar el servicio a clientes afrodescendientes alegando sus creencias religiosas opuestas a la integración racial ${ }^{19}$, y una empresa de fotografía se negó

19 Newman v. Piggie Park Enterprises Inc., 390 U.S. 400. 
a fotografiar la ceremonia de compromiso de una pareja de lesbianas con base en las creencias religiosas de los propietarios de la compañía ${ }^{20}$.

\section{LAS IMPLICACIONES DE HOBBY LOBBY CON RESPECTO A LA TITULARIDAD DE LIBERTAD RELIGIOSA}

Por primera vez en la historia americana, es una persona jurídica con ánimo de lucro y representante de un grupo significativo para la economía del país quien alega una vulneración de su derecho de libertad religiosa. Es importante apuntar que más de ciento sesenta millones de trabajadores prestan sus servicios a corporaciones («Employment Situation Summary», s. f.) y más de dos tercios de esta fuerza laboral es empleada por closely held corporaciones. Por ende, las decisiones que reconocen un derecho a las corporaciones conllevan repercusiones para una gran parte del sector empresarial del país. Quizá esa puede ser una de las razones que expliquen la cantidad y variedad de voces que se han manifestado en contra del fallo, aludiendo a presiones económicas e incluso calificado por algunos como la continuación — después del caso Citizens United - del reconocimiento «desproporcional» de un derecho fundamental para una corporación (Marpe, 2015; Lyle, 2016). Sin embargo, Hobby Lobby es un fallo con un mosaico de temas a los que la Corte va respondiendo para construir finalmente una sentencia que, sin ignorar los otros temas implicados, pretende ser coherente con la libertad de comercio y la libertad de conciencia.

\subsection{El reconocimiento de derechos fundamentales a una corporación closely held}

El caso Hobby Lobby establece uno de los principios fundamentales para construir una teoría sobre el goce de derechos fundamentales por personas jurídicas: una corporación es simplemente una forma de organización utilizada por personas para lograr fines en común. Cuando los derechos fundamentales se extienden a las corporaciones el propósito es proteger los derechos de sus socios, por tanto, sus derechos derivan de los derechos individuales de sus accionistas, ya que, como ficción legal, es indispensable la intervención de sus representantes para decidir y responder a sus derechos y obligaciones. Esto implica, a su vez, que la titularidad de derechos por parte de una corporación no puede ser superior a los derechos de sus accionistas (Werhane, 1985: 61).

En Hobby Lobby la forma organizativa — closely held — pesa considerablemente en la resolución del caso, hasta el punto de ser la estructura de la

20 Elane Photography, LLC v. Willock, 309 P.3d 53. 
persona jurídica un elemento indispensable a la hora de determinar la existencia de una "conciencia empresarial». Esta se puede entender como un núcleo central de valores que identifican a la empresa y es fruto de la voluntad de sus accionistas. En una corporación organizada como closely held es usual la existencia y correspondencia de intereses comunes entre los accionistas debido, principalmente, al limitado número de socios. Por el contrario, las corporaciones con acciones cotizables en bolsa tienen el reto de contar con una gran cantidad de accionistas —incluso de diferentes procedencias geográficas y creencias religiosas-, y su fin lucrativo suele ser el primordial. A modo de ejemplo, una empresa como la cadena de supermercados Walmart, con más de dos millones de trabajadores, con una dificultad real de reunir a todos sus accionistas en un mismo lugar y con un eslogan comercial marcadamente lucrativo (Walmart, s. f.), presenta un escenario más complejo para considerar el planteamiento de una demanda judicial ante la violación de sus creencias religiosas.

\subsection{Las corporaciones con ánimo de lucro y la libertad religiosa}

La Corte hace una breve consideración sobre las personas jurídicas y recurre al Dictionary Act para catalogar a las corporaciones bajo la condición de «persona». La Corte no analiza con detalle la cuestión de la titularidad por la persona jurídica, sino que, por el contrario, analiza la viabilidad de hacer diferencias entre personas jurídicas cuyo fin sea el lucro o no. De acuerdo con la Administración, y varios amicus curiae contrarios (Nejaime y Siegel, 2015), las corporaciones sin ánimo de lucro están asociadas con fines altruistas y el lucro es un elemento secundario, por tanto, la misión realizada por una iglesia y por una corporación con ánimo de lucro puede ser similar en cuanto medios, pero sus fines son completamente distintos. Sostienen que es justificable utilizar el criterio del lucro como un elemento diferenciador en razón de desvincular el poder económico del poder religioso para evitar desequilibrios en las estructuras sociales y en el acceso a la justicia ${ }^{21}$.

Los anteriores argumentos parecen ser débiles al presentar el lucro y la práctica de una religión como incompatibles. Si bien el ejercicio religioso por

21 Este argumento resuena en otros organismos internacionales, como la Corte Interamericana de Derechos Humanos, donde se excluye el reconocimiento de los derechos humanos a personas jurídicas para evitar posibles desequilibrios. Para una posición contraria y una revisión exhaustiva del debate, consúltese: opinión consultiva OC-22/16: titularidad de derechos de las personas jurídicas en el Sistema Interamericano de Derechos Humanos, 26 de febrero de 2016 y De Casas y Toller (2015). 
parte de una persona física se suele manifestar con actos externos — indumentaria, visita a lugares de culto público, etc.-, la persona jurídica puede realizar ciertos actos que la identifiquen con una determinada fe. Los amicus curiae a favor respaldan que una corporación con ánimo de lucro se constituya con el objetivo de proteger o difundir los valores religiosos o morales de sus accionistas a través de la administración de su negocio. Siguiendo la opinión de J. E. Dunn Construction Group Inc., los valores religiosos impregnan todos los aspectos de la vida de quienes creen en ellos, llevándolos en un ámbito lucrativo a formar empresas que representan y promueven los valores que son fundamentales para su fe (Corte Suprema de Estados Unidos, 2013c: 16-21); de igual manera, el Cato Institute entiende la fe como un ámbito de la vida de las personas que condiciona los otros aspectos de su vida: su vida social, laboral, etc. (Corte Suprema de Estados Unidos, 2013d: 16-21).

Los dueños de Hobby Lobby entienden su negocio como una «vocación» que les permite expandir sus valores religiosos. Si bien es cierto que las corporaciones "no tienen ni conciencia, ni creencias, ni sentimientos, ni pensamientos, ni deseos» (Johnson, 2012: 1141), debido a que solo los seres humanos rezan, acuden a los cultos religiosos y pueden creer en un ser superior —además de la imposibilidad física de hacerlo—, también es cierto que una corporación tiene ideario, valores, misión, cultura empresarial, etc., que la definen e identifican en la manera en que conduce su negocio. De esta forma, una corporación puede plantearse el apoyo a otros temas no necesariamente relacionados con su giro comercial pero que considere que poseen un interés para intervenir.

Pensamos que la responsabilidad social corporativa es un ejemplo que puede ayudar a explicar el argumento anterior; esta práctica es una filosofía y una actitud empresarial proyectada en la búsqueda equilibrada de la rentabilidad económica, la mejora del bienestar social de la comunidad y la preservación del medio ambiente (Fernández, 2016: 19). Este ideario parte del reconocimiento de la empresa no solo como una máquina lucrativa, sino como una organización de personas con impacto en el medio y que, por ende, puede influenciar en él con el objeto de mejorarlo de acuerdo con sus valores. Por tanto, si los administradores o accionistas de una empresa pueden expresar sus valores e ideología en la reducción de la contaminación, la mejora de las normas laborales y el cambio climático, no es coherente excluir a las creencias religiosas (Cioccheti, 2014: 52).

Para lograr impulsar una política empresarial de responsabilidad social corporativa o un ideario religioso, siguiendo con los principios básicos del derecho societario, es importante que las decisiones sean tomadas por una mayoría de socios, y por ende, representen la voluntad de la corporación. Siguiendo la opinión presentada por el amicus curie Knights of Columbus, el 
ideario religioso de una corporación con ánimo de lucro puede manifestarse por diversos medios: los votos de los accionistas, los documentos donde se establecen la misión y valores de una empresa, los manuales y guías para los empleados, así como la forma en que la empresa lleva a cabo sus operaciones día a día, e incluso las creencias religiosas de sus clientes (Corte Suprema de Estados Unidos, 2013e: 22-24). Cuestión distinta es qué concretas manifestaciones del actuar de la persona jurídica pueden considerarse incluidas en la libertad religiosa.

\subsection{El rol del Estado ante la libertad religiosa y las corporaciones}

El Gobierno no puede obligar a sus ciudadanos a renunciar a sus derechos de libre ejercicio al administrar un negocio, elegir una institución para cursar sus estudios o aceptar un puesto de trabajo. Ni la jurisprudencia de la Corte ni la Ley de Restauración de la Libertad Religiosa justifican la renuncia de las creencias religiosas de los individuos en el momento de organizarse bajo una persona jurídica (Corte Suprema de Estados Unidos, 2013d: 4). En la economía moderna las corporaciones aportan inmensas facilidades para la ejecución de los negocios; ser coherente con un ideario religioso puede suponer una gran desventaja competitiva ya que o se sigue el modelo lucrativo empresarial y se dejan de lado las creencias religiosas o se utiliza una figura menos competitiva — por ejemplo, sin ánimo de lucro— con tal de ser coherente con los valores religiosos.

Las acciones gubernamentales que vulneran la libertad religiosa de una persona jurídica deben evitar colocar a los accionistas en una posición donde se vean forzados a elegir entre mantener sus convicciones religiosas o abandonarlas. Esto conllevaría que, al crear un negocio, el ciudadano partiese de la posibilidad de que el Gobierno obligase a su empresa a tomar medidas contrarias a su fe; por ejemplo, una corporación constituida y administrada por musulmanes podría verse obligada a participar en transacciones financieras que son gharar; o a una de testigos de Jehová podría imponérsele la producción de armamento, o una panadería judía se le podría negar la venta de pan kosher (Corte Suprema de Estados Unidos, 2013d: 18); ejemplos similares se podrían señalar en relación no con una religión, pero sí con una ideología o con determinados valores. Esto evidencia que la tutela del derecho para la persona legal es necesaria para la tutela de los derechos fundamentales de las personas físicas que están detrás.

El Gobierno debe de asegurarse de que la libertad religiosa no se convierta en un vehículo que legitima radicalismos o permita discriminaciones ilegales e irrazonables. Se entiende que a raíz de los derechos de propiedad que poseen los accionistas, y siempre que se respeten los derechos de los 
trabajadores, pueden administrar su negocio de la manera que consideren oportuna, dictando determinadas reglas que pueden abarcar aspectos de vestimenta hasta forma de comportarse (Strine, 2015). No obstante, aquellas disposiciones que involucren una manifestación religiosa en el lugar de trabajo o incidan en general en algún derecho constitucional de los trabajadores deben ser manifestadas tanto en el momento de realizar una oferta laboral como en el momento en el que se vayan a implementar en la empresa.

Hobby Lobby v. Burwell supuso la incorporación de nuevos argumentos al debate sobre la titularidad de los derechos fundamentales de las personas jurídicas en Estados Unidos. Con la conformación actual de la Corte Suprema, y la incorporación del juez Neil Gorsuch, no son de esperar mayores cambios en el debate, caracterizado por su abordaje pragmático y antiteórico. Con respecto al Obamacare, la Administración del presidente Donald Trump manifestó, desde la campaña electoral, su intención de eliminar dicha ley; actualmente se encuentra en proceso de sustitución.

\section{Bibliografía}

Alliance Defending Freedom (2014). Brief for petitioners. Disponible en: https://bit.ly/2JC$\mathrm{mUs} 3$.

Barnett, J. y Vornovitsky, M. (2015). Report Number: P60-257. Health Insurance Coverage in the United States: 2015, United States Census Bureau. Disponible en: https://bit.ly/2cl2nsj.

Benson, R. (1998). The Need for and Cost of Mandating Private Insurance Coverage of Contraception. Disponible en: https://bit.ly/2O09QPS.

Black, H. C. (2011). Black's law dictionary (4. 'ed.). New York: Thomson Reuters.

Blair, M. M. (2013). Corporate Personhood and the Corporate Persona. University of Illinois Law Review, (3), 785-820.

Brook, T. (1991). The New Historicism. New Jersey: Princeton University Press.

Brown, T. (2013). In-Corp-O-Real: A Psychological Critique of Corporate Personhood and Citizens United. Florida State University Business Review, 12 (1), 1-108.

Bulling, R. (2011). Tom the dancing bug: Hey, Kids! The World You're On... has CORPORATIONS! Boing boing. Disponible en: https://bit.ly/2uA5CXX.

Cioccheti, C. (2014). Religious Freedom and Closely Held Corporations: The Hobby Lobby Case and Its Ethical Implications. Oregon Law Review, 93 (2), 259-354

Clancy, T. (2010). The Fourth Amendment as a Collective Right. Texas Tech Law Review, 43 (1), 255-298.

Corte Suprema de Estados Unidos (2013a). Brief for respondents. Disponible en: https://bit. ly/2O38vso. 
(2013b). Brieffor petitioners. Disponible en: https://bit.ly/2zTHHrt.

(2013c). Brieffor the J.E Dunn Construction Group Inc., as amicus curiae in support of the Hobby Lobby respondents and the Conestoga petitioners. Disponible en: https://bit. ly/2uUTGzx.

- (2013d). Brieffor the Cato Institute as amicus curiae in support of the Hobby Lobby respondents and the Conestoga petitioners. Disponible en: https://bit.ly/2Lc17gI.

- (2013e). Brief for the Knights of Columbus as amicus curiae in support of the Hobby Lobby respondents and the Conestoga petitioners. Disponible en: https://bit.ly/2uFGPBZ.

- (2014). Opinion of the Court, 573 U. S. (2014). Disponible en: https://bit.ly/2ozQPcS. Cox, J. (2003). Corporations. New York: ASPEN.

De Casas, I. y Toller, F. (2015). Los derechos humanos de las personas jurídicas. México: Editorial Porrúa.

De Salas Murillo, S. (2011). Capítulo II. De las personas jurídicas. En Código Civil comentado. Navarra: Editorial Aranzadi.

Dewey, J. (1926). The Historic Background of Corporate Legal Personality. Yale Law Journal, 35 (6), 656-673. Disponible en: https://doi.org/10.2307/788782.

DeWitt, L. (2010). The Development of Social Security in America. Social Security Bulletin, 70 (3), 1-26.

Díez-Picazo, L. y Gullón, A. (2012). Sistema de Derecho Civil (vol. I.). Madrid: Tecnos.

Donald J. Trump for President. (s. f.). Disponible en: https://www.donaldjtrump.com

Eberle, E. J. (2003). The territorial and personal scope of fundamental rights in United States of America. Fundamental rights in Europe and North America. Leinden: Brill Academic Publishers.

Employment Situation Summary. (s. f.). Disponible en: http://bit.ly/2vjaC2M.

Executive Order Minimizing the Economic Burden of the Patient Protection and Affordable Care Act Pending Repeal (2017, 20 de enero). Disponible en: http://bit.ly/2OwgVIX.

Feinstein, D. (2009). Statement of Senator Dianne Feinstein in Support of the Mikulski Amendment \#2791. Disponible en: https://bit.ly/2v4QUsc.

Fernández, R. (2016). Responsabilidad social corporativa: una nueva cultura empresarial. Alicante: Editorial Club Universitario.

Garrett, B. (2014). The constitutional standing of corporations. University of Pennsylvania Law Review, 163 (1), 96-162.

Gaughan, M. (2012). Corporations are not People: An Analysis of Citizens United v. Federal Election Commission. University of Puget Sound, 143 (2), 1-33.

Gedicks, F. M. (2017). Substantial Burdens: How Courts May (and Why They Must) Judge Burdens on Religion Under RFRA. George Washington Law Review, 85 (1), 94-151.

Gobierno de Estados Unidos (2013). Coverage of certain preventive services under the Affordable Care Act. Disponible en: https://bit.ly/2zSeRaT.

Gobierno de Estados Unidos (s. f.). HHS historical highlights: the U. S. Department of Health and Human Services. Disponible en: https://bit.ly/2Ntc5e9.

Gómez Montoro, Á. J. (2002). La titularidad de derechos fundamentales por personas jurídicas: un intento de fundamentación. En Libro homenaje al profesor Francisco Rubio Llorente. Madrid: Centro de Estudios Políticos y Constitucionales. 
Hovenkamp, H. (1988). The classical corporation in american legal thought. Georgetown Law Journal, 76 (2), 1593-1650.

Institute of Medicine of The National Academies (2011). Clinical Preventive Services for Women: Closing the Gaps at 1-2. Disponible en: https://bit.ly/2n2Ra6I.

Johnson, L. (2012). Law and legal theory in the history of corporate responsibility: corporate personhood. Seattle University Law Review, 35 (1135), 1135-1164.

Kane, D. (1977). The Applicability of the Double Jeopardy Right to Corporations. Duke Law Journal, 26 (3), 726-750. Disponible en: https://doi.org/10.2307/1371958.

Kollmann, G. (1996). Summary of Major Changes in the Social Security Cash Benefits Program: 1935-1996. Congressional Research. Disponible en: http://bit.ly/2LCntb3.

Krannich, J. (2005). The corporate "person": A new analytical approach to a flawed method of constitutional interpretation. Loyala University Chicago Law Journal, 37 (1), 61-109.

Kutoroff, A. (2015). First Amendment versus Laicité: Religious exemptions, religious freedom, and public neutrality. Cornell International Law Journal, 48 (1), 247-282.

Loewentheil, K. (2014). When free exercise is a burden: protecting third parties in religious accommodation law. Drake Law Review, 2 (62), 433-502.

Lyle, J. (2016). Contraception and Corporate Personhood: Does the Free Exercise Clause of the First Amendment Protect for-Profit Corporations that Oppose the Employer Mandate. University of Dayton Law Review, 39 (1), 137-172.

Magrath, P. (1963). Morrison R. Waite: The triump of character. New York: Macmillan.

Marín López, J. J. (2009). De las personas jurídicas. En R. Rodríguez-Cano Bercovitz (dir.). Comentarios al Código Civil (cap. 2). Pamplona: Aranzadi.

Mark, G. A. (1987). The personification of the Business Corporation in American Law. University of Chicago Law Review, 54 (4), 1441-1483. Disponible en: https://doi. org/10.2307/1599739.

Marpe, L. M. (2015). Craft store, corporation, and birth control: why the hobby lobby decision is logical extension for corporate personhood and how its narrow holding will mitigate the potential impact. UMKC Law Review, 84 (2), 519-542.

Mayer, C. J. (1989). Personalizing the impersonal: corporations and the Bill of Rightst. Hastings Law Journal, 41 (1), 577-668.

McFadden, B. (2011). The strip. New York Times, 20-8-2011. Disponible en: https://nyti. $\mathrm{ms} / 2 \mathrm{~L} 8 \mathrm{~N} 1 \mathrm{wm}$.

McBride, D. (2011). General Corporation Laws: History and Economics. Law and Contemporary Problems, 74 (1), 1-18.

Morrison, A. B. (2008). Fundamentals of American law. New York: Oxford University Press.

Nace, T. (2003). Gangs of America. San Francisco: Berett-Koehler.

National Women's Law Center. (2015). Status of the Lawsuits Challenging the Affordable Care Act's Birth Control Coverage Benefit. Disponible en: https://bit.ly/2uHWAZ9.

Nejaime, D. y Siegel, R. (2015). Conscience Wars: Complicity-Based Conscience Claims in Religion and Politics. Yale Law Journal, 124 (7), 2516-2591.

Nicholson, B. (1992). The Fiduciary Duty of Close Corporation Shareholders: A Call for Legislation. American Business Law Journal, 30 (3), 513-536. Disponible en: https://doi. org/10.1111/j.1744-1714.1992.tb00666.x. 
O'Kelly, C. (1979). The Constitutional Rights of Corporations Revisited: Social and Political Expression and the Corporation After First National Bank v. Bellotti. The Georgetown Law Journal, 67 (6), 1347-1384.

Pollman, E. (2011). Reconceiving Corporate Personhood. Utah Law Review, 4, 1629-1676.

Rall, T. (2016). Let freedom ring! For abstract legal entities. Disponible en: http://bit. ly/2NWRO0G.

Reyes Villamizar, F. (2006). Derecho societario en Estados Unidos. Introducción comparada. Colombia: Legis.

Ripken, S. K. (2011). Citizens United, Corporate Personhood, and Corporate Power: The Tension between Constitutional Law and Corporate Law. University of St. Thomas Journal of Law and Public Policy, 6 (2), 285-316.

Romano, R. (1987). The State Competition debate in Corporate Law. Cardozo Law Review, 8 (4), 709.

Strine, L. (2015). Job Is Not a Hobby: The Judicial Revival of Corporate Paternalism and Its Problematic Implications. Journal of Corporation Law, 41 (1), 71-116. Disponible en: https://doi.org/10.2139/ssrn.2555816.

United States Court of Appeals for The Tenth Circuit. (2013). Precedendial: Hobby Lobby $v$. Burwell. Disponible en: https://bit.ly/2JFIz2C.

- (2013). Precedendial: Conestoga v. Burwell. Disponible en: https://bit.ly/2mto8wu.

- (2013). About the ACA. Disponible en: https://bit.ly/2oHr3kh.

Vargas Vargas, M. (1964). La sociedad anónima en el derecho anglonorteamericano. Santiago: Editorial Jurídica de Chile.

Villegas García, M. Á. (2016). La responsabilidad criminal de las personas jurídicas. La experiencia de Estados Unidos. Navarra: Thomson Reuters.

Wallmart (s. f.). Our Business. Disponible en: https://bit.ly/1OqLSs7.

Werhane, P. (1985). Persons, rights, and corporations. New Jersey: Prentice-Hall.

Wood, S. G. y Scharffs, B. G. (2002). Applicability of Human Rights standards to private corporations : an american perspective. The American Journal of Comparative Law, 50 (3), 531-566. Disponible en: https://doi.org/10.1093/ajcl/50.suppl1.531. 